\title{
Anticipation of incentive gain
}

\author{
CHARLES F. FLAHERTY and SUSAN CHECKE \\ Rutgers University, New Brunswick, New Jersey
}

\begin{abstract}
In four experiments, the once daily availability of saccharin $(.15 \%)$ preceded the availability of sucrose $(32 \%$ or $2 \%)$. Experiment 1 showed that the intake of saccharin was reduced when it preceded $32 \%$ sucrose but not when it preceded $2 \%$ sucrose, as compared with saccharin-alone conditions. Experiment 2 showed that less saccharin was consumed when the saccharin preceded sucrose by $5 \mathrm{~min}$ than when there was a 30-min intersolution interval. Experiment 3 replicated this finding and showed that the presentation of the two solutions through the same or different access holes in the apparatus was not relevant to the result. Experiment 4 showed that there was an inverse relationship between saccharin intake and the length of the intersolution interval in the range of 1 to $30 \mathrm{~min}$. These data were interpreted to indicate that the animals learn the predictive relationship between the saccharin and sucrose solutions and that the intake of the saccharin is reduced by an anticipatory contrast mechanism-a mechanism that may have restricted temporal parameters.
\end{abstract}

The juxtaposition of sucrose solutions of high and low concentration leads to a reduced intake of the solution of lower concentration compared with conditions in which only the lower concentration is experienced. This diminished intake occurs when the lower concentration is presented after the animal has had extended or minimal experience with the higher concentration (Flaherty, Ciszewski, \& Kaplan, 1979; Flaherty, Troncoso, \& Deschu, 1980; Vogel, Mikulka, $\&$ Spear, 1968), as well as when the two solutions are repeatedly alternated in availability (Flaherty \& Largen, 1975; Flaherty \& Sepanak, 1978).

The diminished intake is termed a negative contrast effect-successive negative contrast when the lower concentration is presented after some experience with the higher concentration, and simultaneous negative contrast when the two solutions are repeatedly juxtaposed. There is some evidence that these two types of contrast may be controlled by somewhat different mechanisms (e.g., Flaherty, Lombardi, Wrightson, \& Deptula, 1980).

Recently we have found indications of another type of contrast, anticipatory contrast (Flaherty \& Checke, Note 1). In the course of a study investigating possible conditioning of the glucoregulatory system with saccharin as a CS and sucrose as a UCS, we saw some evidence that the intake of saccharin was reduced when it served as a predictor of sucrose. The studies described in this paper explored this phenomenon.

This research was supported by a grant from the Rutgers Research Council. Address correspondence to Charles Flaherty, Psychology Department, Busch Campus, Rutgers University, New Brunswick, New Jersey 08903.

\section{EXPERIMENT 1}

The purpose of the first experiment was to determine whether the presentation of a sucrose solution 5 min after the daily availability of a $.15 \%$ saccharin solution would lower the intake of saccharin. A second purpose was to determine whether the concentration of the sucrose solution was important in determining the degree of suppression of saccharin.

The basic procedure in this experiment, as well as in most of the other experiments in this paper, was as follows. A tube containing a $.15 \%$ saccharin solution was made available for a 3-min period beginning with the rat's first lick. At the end of this period, the tube was withdrawn. After the passage of a time interval ( $5 \mathrm{~min}$, in this experiment), a second tube containing a sucrose solution was presented for a 5-min period, beginning with the first lick. Control animals received the saccharin but no sucrose and were left in the apparatus for as long as the animals receiving the sucrose. In the present experiment, one group of animals received a $32 \%$ sucrose solution on each day following saccharin, a second group received a $2 \%$ sucrose solution.

\section{Method}

Subjects. The subjects were 23 Sprague-Dawley male rats weighing 428-671 $\mathrm{g}$ that had had previous experience in an insulin conditioning study. Twenty-one of the 23 also had had experience in an operant barpressing experiment with Noyes pellet reinforcements. They had had no prior experience with saccharin or sucrose. All rats were housed individually on a 14/10 light-dark cycle. The rats were maintained at $82 \%$ of their free-feeding weights by onceper-day feeding. Water was available continuously in the home cage.

Apparatus. Animals were tested in two clear Plexiglas chambers measuring $30 \times 25 \times 25 \mathrm{~cm}$. Two $1.5-\mathrm{cm}$ holes had been drilled in one side of each box, $21.7 \mathrm{~cm}$ apart and $4 \mathrm{~cm}$ from the wiremesh floor. Solutions were delivered via motors that could ad- 
vance or withdraw graduated cylinders to align their drinking tubes with these openings.

Procedure. Subjects were assigned to one of three groups counterbalanced for weight. Groups were sacch-only $(n=7)$, sacch $+2 \%$ $(n=8)$, and sacch $+32 \%(n=8)$. One trial per day was given for 12 consecutive days. In each group, the saccharin tube was presented at one hole (right) for $3 \mathrm{~min}$ and then withdrawn for an inter$\mathrm{val}$ of $5 \mathrm{~min}$. At the end of this interval, either $2 \%$ or $32 \%$ sucrose was made available at the second (left) hole for $5 \mathrm{~min}$. The sacchonly group was left in the apparatus for an additional $5 \mathrm{~min}$ at this time with no solution present. Licks were counted with a contact relay circuit, and all solution availability periods were timed from the first lick.

The running order of the animals was sacch-only, sacch $+2 \%$, and sacch $+32 \%$. Sucrose tubes were removed when the sacchonly group was run, and the apparatus was wiped with a damp sponge between trials to control for spillage.

A . $15 \%$ saccharin solution was used. Sucrose was $2 \%$ or $32 \%$ by weight (sucrose/sucrose + water) and was mixed $24 \mathrm{~h}$ prior to use from commercial-grade cane sugar and tap water.

\section{Results}

The mean licks on the saccharin solution for each group are presented as a function of days in Figure 1. It is apparent that the groups that received saccharin only or saccharin followed by $2 \%$ sucrose showed a substantially more accelerated lick function than did the animals for whom the saccharin was followed by $32 \%$ sucrose. A reliable groups $\times$ days term $[F(20,201)$ $=2.06, \mathrm{p}<.01]$ from analysis of variance followed by least significant difference (LSD) tests indicated the following pattern. The saccharin-only group had a reliably higher lick frequency than the saccharin-32\% sucrose group on Days 6-11, and the saccharin-2\% sucrose group had a higher lick frequency than the saccharin-32\% group on Days 5-7 and 9-10. The saccharin-2\% sucrose group was never reliably below the saccharin-only group.

An analysis of the lick data for the sucrose solu-

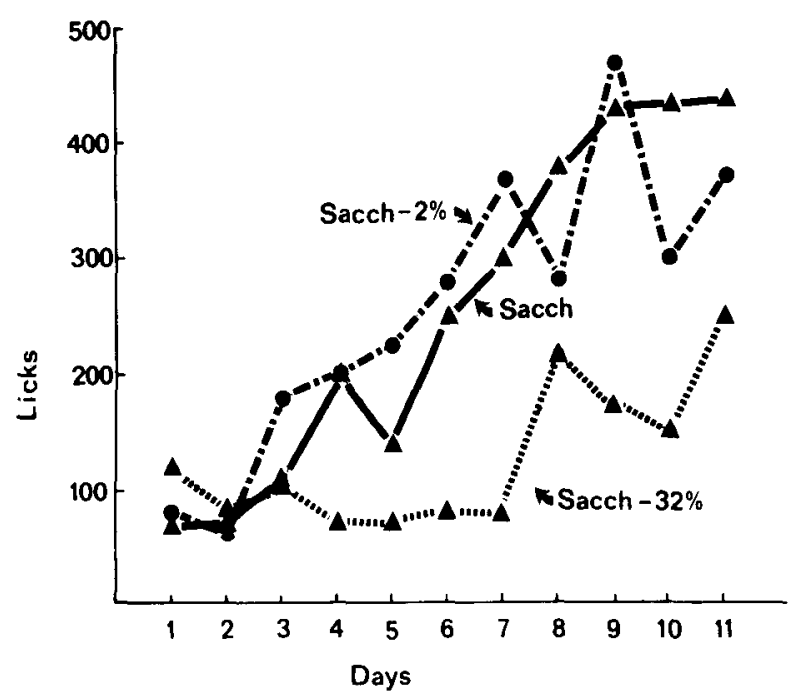

Figure 1. Lick frequency for saccharin as a function of following sucrose condition ( (sacch $=$ no following sucrose). tions indicated that the group receiving $32 \%$ sucrose licked at substantially higher rates than did the $2 \%$ rats [Day 12 mean licks $/$ min for $2 \%$ sucrose $=34$, mean licks $/ \mathrm{min}$ for $32 \%$ sucrose $=268, F(1,14)=$ $32.18, \mathrm{p}<.01$ ]. Mean terminal licks/min for saccharin was 150 .

\section{Discussion}

These results show that the increase in saccharin intake that normally occurs as a function of experience with saccharin is suppressed when that saccharin is followed after $5 \mathrm{~min}$ by $32 \%$ sucrose, but not when the saccharin is followed by $2 \%$ sucrose. It is reasonable to consider the suppression of the saccharin intake as a form of contrast since $32 \%$ sucrose has considerably greater hedonic value than $.15 \%$ saccharin, as is evident in the lick frequencies obtained in this experiment (see also Collier \& Novel, 1967, and Young $\&$ Madsen, 1963). Two percent sucrose does not have greater hedonic value than $.15 \%$ saccharin-also seen in the lick frequencies obtained in this experiment and in the data of Pfaffman (1960) and Young and Madsen (1963). Previous research has shown that contrast effects obtained in the consumption of different concentrations of sucrose solutions parallels the relative hedonic value of the solutions (Flaherty \& Kaplan, 1979; Flaherty \& Sepanak, 1978).

If the suppression of saccharin intake does represent a form of contrast, then the question arises as to how the rats make the comparison between the two solutions. That is, is the contrast anticipatory in nature-is saccharin intake suppressed because of the impending sweeter sucrose-or is the contrast due simply to the fact that the rats had received the sweeter sucrose on previous days in that same apparatus? The remaining experiments are addressed to this question.

\section{EXPERIMENT 2}

Successive negative contrast effects routinely occur with $24 \mathrm{~h}$ separating the preshift from the postshift solution (e.g., Flaherty et al., 1980), even if the animals have had minimal experience with the preshift solution (Flaherty et al., 1979). Thus, it is possible that the suppression of saccharin intake each day results from a comparison of the saccharin with the sweeter sucrose solution received some $24 \mathrm{~h}$ earlier. If this successive comparison were the basis of the saccharin suppression, then manipulating the time between saccharin and sucrose solutions within a day would be expected to have little or no effect on contrast.

On the other hand, if saccharin suppression resulted from anticipation of the impending $32 \%$ sucrose, then lengthening the time between saccharin and sucrose might be expected to reduce the degree of contrast occurring in saccharin intake. This factor 
was investigated here in groups of rats for whom the time between saccharin and $32 \%$ was either 5 or $30 \mathrm{~min}$. The intake of saccharin in these groups was compared with that in animals that experienced saccharin only.

\section{Method}

Subjects. Eighteen naive, male rats derived from the SpragueDawley strain were used as subjects. The rats were deprived to $82 \%$ of their $355-415-\mathrm{g}$ free-feeding weights and were maintained at that level by once-per-day feedings. The rats were housed individually on a $14 / 10$ light-dark cycle with water available continuously.

Apparatus. The apparatus was the same as that used in Experiment 1 .

Procedure. The rats were divided randomly into three groups $(n=6)$. The first group received 3-min access to saccharin, then a 30-min interval with no solution available, and then 5-min access to $32 \%$ sucrose (Group Sacch-30-Suc); the second group received a 5-min interval between the saccharin and sucrose solutions (Group Sacch-5-Suc), and the third group received saccharin only (Group Sacch). This latter group was actually divided into two subgroups, one of which was left in the apparatus for $10 \mathrm{~min}$ following saccharin consumption and one of which was left in for 35 min-times approximately equivalent to those experienced by the two sucrose groups. This differential stay in the apparatus did not appear to affect saccharin consumption in these control animals, and, thus, the two subgroups were combined for data analysis.

One trial (exposure to the solutions) was presented each day for 8 days. The sucrose and saccharin tubes were presented through different holes (left and right) in the apparatus, and the inside of the apparatus was wiped down with water between successive rats. In this experiment, the sacch group was run each day before any of the sacch-sucrose groups. The sucrose and saccharin were mixed as in Experiment 1.

\section{Results}

One rat in the saccharin-only group was dropped from the experiment for failure to lick the saccharin solution. The mean lick frequency obtained from the remaining animals is presented in Figure 2 . It is apparent that the lick frequency of the animals for whom saccharin preceded sucrose was lower than that of the animals that received only saccharin. Furthermore, the rats with a 5-min interval between the two solutions licked less saccharin than did the rats with a 30-min break between solutions. These

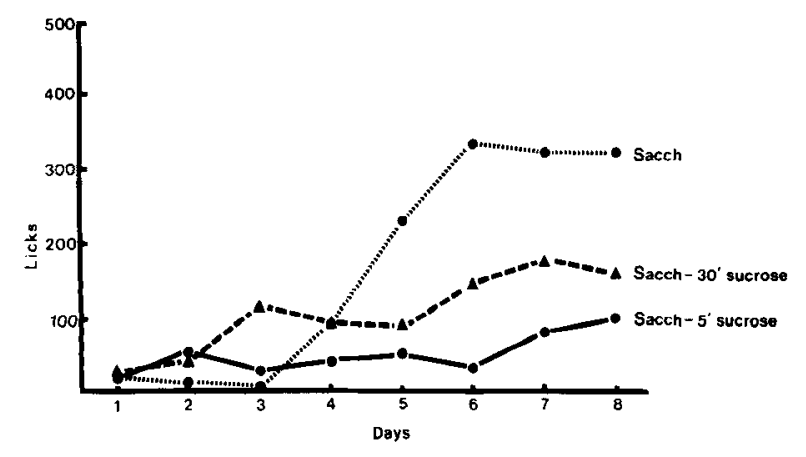

Figure 2. Lick frequency for saccharin alone or saccharin followed 5 or $30 \mathrm{~min}$ later by $32 \%$ sucrose. differences among the three groups were statistically reliable [groups main effect, $F(2,14)=19.38, p<.01$, followed by LSD tests with $\mathrm{p}=.05]$.

Further examination of the daily means showed that the lick frequency of the sacch group was greater than the sacch-5-suc group on Days 4-8 and greater than the sacch-30-suc group on Days 5-8. The sacch30 -suc group had a higher lick frequency than the sacch-5-suc group on Days 3 and 6-8. Finally, the sacch-30-suc group had a higher lick frequency than the sacch group on Day 3. All of these reliability statements are based on a significant group $\times$ days term $[F(14,96)=4.37, p<.01]$ followed by LSD tests $(\mathrm{p}=.05)$.

The sacch-30-suc and sacch-5-suc groups did not differ in their lick rate for the $32 \%$ sucrose solution $(\mathrm{F}<1)$.

\section{Discussion}

The suppression of the saccharin intake was greater when there were only 5 min between the saccharin and the subsequent sucrose solution than when there were $30 \mathrm{~min}$ between the two solutions. If the suppression in saccharin intake was due to the comparison of the saccharin with the sucrose received on the previous day, as in successive contrast, then the interval between the saccharin and sucrose within a day would be expected to have little or no effect. In fact, to the extent that the longer within-day interval reduced the normal 24-h between-day comparison interval, it would be expected that contrast would be enhanced. Thus, the finding of reduced contrast with the 30-min within-day interval supports the hypothesis that the reduced saccharin intake is due to the anticipation of the impending sucrose rather than to the fact that sucrose was received $24 \mathrm{~h}$ earlier.

\section{EXPERIMENT 3}

Experiments 1 and 2 provided evidence that saccharin intake is suppressed by the availability of a highly concentrated sucrose solution a short time after the saccharin is withdrawn. The present experiment was concerned with whether the saccharin suppression was an artifact of the manner in which the two solutions were presented. In both previous experiments, the two solutions were presented through different openings in the apparatus. It is possible that, despite the wipings between animals, there was sufficient spillage of the sucrose for the animals to obtain samples of the sucrose from the walls or grid floor of the apparatus in the vicinity of the sucrose tube. If the animals that received both solutions, but not the saccharin-only animals, learned the location of such sucrose and obtained sample tastes while the saccharin was present, then this could account for the suppression in saccharin intake without the necessity of the concept of anticipation. 
Although this possibility seems quite unlikely and does not account for the temporal effect, it is still reasonable to attempt to rule it out as an explanation. For this purpose, both saccharin and sucrose solutions were presented through the same opening in the center of the apparatus for all animals. It was thought that this modification would make any spillage not removed by the wipe down equally available to all animals.

\section{Method}

Subjects. Nine female and eight male Sprague-Dawley rats were used as subjects. Fourteen of the rats had had prior experience in either a spatial learning or a food intake study. They were deprived to $82 \%$ of their free-feeding weights and were maintained as in the previous studies.

Apparatus. The apparatus was the same as that used in the previous experiments except that an additional $1.5-\mathrm{cm}$-diam hole was drilled halfway between the holes used to present the sucrose and saccharin in the earlier experiments. Both tubes were mounted so as to be available through this central hole when they were inserted for access.

Procedure. The rats were assigned to three groups balanced for prior experience and sex. The groups were saccharin only $(n=6)$, saccharin/5-min interval/sucrose $(n=5)$, and saccharin/ 30-min interval/sucrose $(n=6)$. The procedures were the same as in Experiment 2 except that both solutions were presented through the central access hole, the animals were run for 12 days instead of 8 , the running order was randomized, and a sucrose tube was in position but was never made available to the saccharin-only animals.

\section{Results}

Three animals were dropped from the experiment for failure to drink the solutions; two were from the saccharin-only group, and one was from the saccharin/ 5-min/sucrose group. All were males.

Analysis of terminal acquisition scores (presented in Figure 3) showed that the saccharin-only animals and the saccharin-30-sucrose animals licked reliably

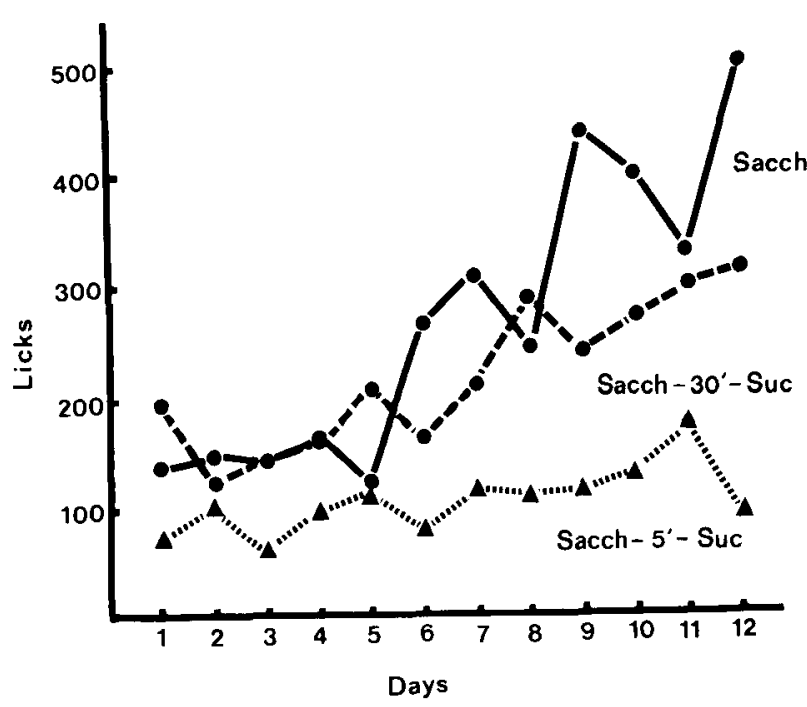

Figure 3. Lick frequency for saccharin alone or saccharin followed 5 or $30 \mathrm{~min}$ later by $32 \%$ sucrose. more for the saccharin than did the saccharin-5sucrose group. The saccharin-only and the saccharin30-sucrose group were not reliably different from each other $[F(2,12)=8.09, p<.01$, followed by LSD tests]. Examination of the day-by-day lick values showed that the saccharin-only and saccharin-30sucrose group diverged from the saccharin-5-sucrose group at about Day 6, the former two groups showing no clear signs of divergence throughout the experiment.

As in the previous experiments, the lick values for the $32 \%$ sucrose solution did not differ between the two groups receiving sucrose $(F<1)$.

\section{Discussion}

The suppression of saccharin intake in the rats given $32 \%$ sucrose $5 \mathrm{~min}$ after the saccharin solution replicates the results obtained in the earlier experiments and indicates that these results were not artifactually related to the separation of the two solutions to different access holes in the apparatus, to the lack of presence of a sucrose tube when saccharinonly animals were in the apparatus, or to the order in which the animals were run.

In the present experiment, unlike Experiment 2, the rats with a 30-min interval separating the saccharin and sucrose solutions showed no suppression of saccharin intake. The reasons for this difference are not obvious. It could be that anticipation over a 30-min interval is a difficult task, one with substantial individual differences, and that the subject sample in the present experiment was such that it precluded this ability. It could also be that the subjects' prior experience influenced this behavior or that the procedural changes, while not affecting the 5-min group, did influence the 30-min group. A fourth experiment was conducted to investigate this result further.

\section{EXPERIMENT 4}

The purpose of this experiment was to explore a wider range of intersolution intervals and to determine whether any evidence of saccharin suppression could be obtained with a 30 -min interval using the procedures of Experiment 3.

\section{Method}

Subjects. Twenty-four adult, male, naive Sprague-Dawley rats were used as subjects and maintained as in the earlier experiments.

Apparatus. The apparatus was the same as in Experiment 3.

Procedure. The rats were assigned randomly to four groups $(n=6)$ designated by the interval between the saccharin and sucrose solutions. The groups received saccharin only or had 1-, $5-$, or $30-\mathrm{min}$ intervals between saccharin and $32 \%$ sucrose solutions. Other aspects of the procedure were the same as in Experiment 3.

\section{Results}

One rat in the saccharin-only group was dropped for failure to lick the solution. 


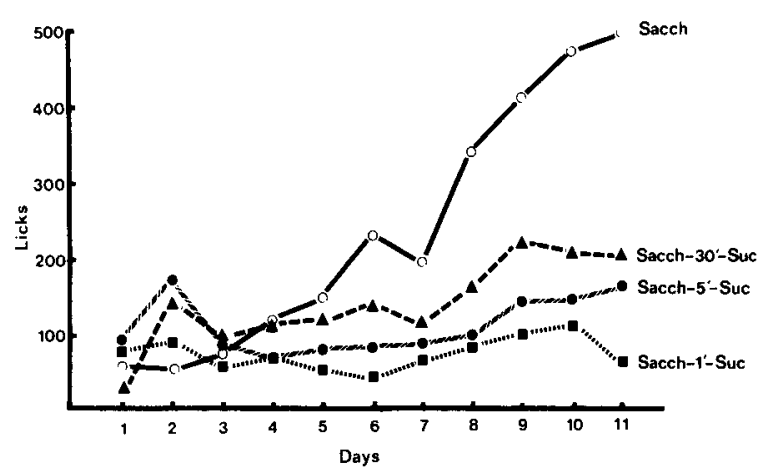

Figure 4. Lick frequency for saccharin alone or saccharin followed 1,5 , or $30 \mathrm{~min}$ later by $32 \%$ sucrose.

Lick frequencies of the four groups as a function of the 11 training days are presented in Figure 4 . It is apparent that all three groups that received sucrose showed a suppressed saccharin intake and that there was temporal ordering in degree of suppression among these groups. The occurrence of this temporal ordering was a reasonable prediction from the previous three experiments and the likelihood that such as ordering would occur by chance is equal to .042 $(=1 / 4 !)$.

The data were also analyzed by a repeated measures analysis of variance, which indicated [groups $X$ days, $F(30,187)=4.24, p<.01]$ that the saccharinonly group had a higher rate than the 1- and 5-min interval groups over Days 6-11 and a greater lick rate than the 30-min group over Days 8-11. The 30-min group licked reliably more than the 1-min group on Days 9 and 11, but was not statistically greater than the 5-min group at any point. Similarly, the consistent difference between the 5- and 1-min groups did not reach statistical reliability.

As in the previous experiments, there was no reliable difference in lick rates for the $32 \%$ sucrose solution among the three groups receiving sucrose.

\section{Discussion}

The present results further substantiate the suppressive effects of impending sucrose on saccharin intake and clearly relate degree of suppression to length of the intersolution interval. The occurrence of a highly reliable suppression in the 30-min group in the present experiment replicates the results of Experiment 2 and indicates that the failure to find suppression with this temporal interval in Experiment 3 was not related to the procedures adopted in that experiment. The possibility remains that the results obtained with the 30-min group in Experiment 3 were a chance finding or perhaps they were related in some way to the fact that the animals had a prior experimental history.

\section{GENERAL DISCUSSION}

These experiments show that sucrose given a short period of time after daily access to saccharin leads to a suppression in saccharin intake. This suppression becomes evident after several daily "pairings," is dependent upon the concentration of the source solution, and varies inversely with the length of the temporal interval between solutions.

Can any mechanism underlying this suppression be specified? Our procedure contains elements of an instrumental learning paradigm in the sense that the presentation of the sucrose is contingent upon at least one lick's being made to the saccharin tube. However, the data show that sucrose presentation in these experiments does not have the effect on saccharin consumption that one would expect from reinforcement of instrumental behavior. For example, it is well established that more highly concentrated sucrose solutions are more reinforcing-acting to increase the rate of behaviors upon which their presentation is contingent (Collier, 1962; Flaherty, Riley, \& Spear, 1973; Guttman, 1953). In Experiment 1, we found that a high concentration of sucrose suppressed saccharin intake relative to a low sucrose concentration. These results are opposite to those expected from a simple application of the law of effect.

The effect of the manipulation of the intersolution interval was also not what would be expected if sucrose were acting as an instrumental reinforcement for saccharin consumption. Delay of reinforcement produces a highly reliable effect on instrumental behavior - the shorter the interval, the higher the rate of instrumental behavior (D'Amato \& Cox, 1976; Davenport, 1962; Logan, 1960; Mackintosh \& Lord, 1973). In our experiments, a direct relationship was obtained between interval length and lick rate for saccharin.

Similarly, it is not reasonable to think of the sucrose as a Pavlovian reinforcer for the licking response, since the lick rate for saccharin is inversely related to the lick rate for sucrose (Experiment 1).

Our interpretation of these data is that the rats learn the predictive relationship (Bindra, 1974; Bolles, 1972) between the saccharin and sucrose solutions, and, once this learning is accomplished, the taste of saccharin serves as a retrieval cue (Chen \& Amsel, 1980; Spear, 1978) for the memory of the ensuing sucrose. Anticipation of the sucrose based on this recall either directly reduces the hedonic value of the saccharin or elicits behaviors that compete with the saccharin consumption (Flaherty, Blitzer, \& Collier, 1978). The effect of the intersolution interval indicates that the contrast is anticipatory in nature rather than being a form of successive or simultaneous contrast. 
These data showing contrast in consummatory behavior augment recent studies indicating the importance of anticipation in other realms of animal behavior, such as sexual responsiveness (Graham \& Desjardins, 1980), avoidance of aversive tastes (Chen \& Amsel, 1980), operant behavior (Williams, 1979, 1981), and runway behavior (Hulse, 1980; Hulse \& Dorsky, 1979). They are also consistent with an earlier study that found that rats fed immediately after an operant session showed suppressed responding in comparison with rats for whom home-cage feeding was delayed for some time after the end of the daily session (Bacotti, 1976).

\section{REFERENCE NOTE}

1. Flaherty, C. F., \& Checke, S. Anticipatory contrast in rats. Paper presented at the meeting of the Psychonomic Society, St. Louis, November 1980.

\section{REFERENCES}

BACotTi, A. V. Home cage feeding time controls responding under multiple schedules. Animal Learning \& Behavior, 1976, 4, 41-44.

Bindra, D. A motivational view of learning performance and behavior modification. Psychological Review, 1974, 81, 199-213.

Bolles, R. C. Reinforcement, expectancy, and learning. Psychological Review, 1972, 79, 394-409.

Chen, J., \& Amsel, A. Recall (versus recognition) of taste and immunization against aversive taste anticipation based on illness. Science, 1980, 209, 831-833.

Collier, G. Consummatory and instrumental responding as a function of deprivation. Journal of Experimental Psychology, $1962,64,410-414$.

Collier, G., \& Novell, K. Saccharin as a sugar surrogate. Journal of Comparative and Physiological Psychology, 1967, 64, 404-408.

D'Aмато, M. R., \& Cox, J. D. Delay of consequences and short term memory in monkeys. In D. Medin, R. Davis, \& W. Roberts (Eds.), Coding processes in animal memory. Englewood Cliffs, N.J: Erlbaum, 1976.

DAVENPoRT, J. W. The interaction of magnitude and delay of reinforcement in spatial discrimination. Journal of Comparative and Physiological Psychology, 1962, 55, 267-273.

Flaherty, C. F., Blitzer, R., \& Collier, G. H. Open field behaviors elicited by reward reduction. American Journal of Psychology, 1978, 91, 429-443.

Flaherty, C. F., Ciszewski, W. A., \& Kaplan, P. S. Retention of taste quality following brief exposure to sucrose. Chemical Senses and Flavour, 1979, 4, 73-78.

Flaherty, C. F., \& Kaplan, P. Gustatory contrast in rats. Chemical Senses and Flavour, 1979, 4, 63-72.
Flaherty, C. F., \& Largen, J. Within subjects positive and negative contrast effects in rats. Journal of Comparative and Physiological Psychology, 1975, 88, 653-664.

Flaherty, C. F., Lombardi, B. R., Wrightson, J., \& Deptula, D. Conditions under which chlordiazepoxide influences gustatory contrast. Psychopharmacology, 1980, 67, 269-277.

Flaherty, C. F., Riley, E. P., \& SPEar, N. E. Effects of sucrose concentration and goal units on runway behavior in the rat. Learning and Motivation, 1973, 4, 163-175.

Flaherty, C. F, \& Sepanak, S. Bidirectional contrast, matching, and power functions obtained in sucrose consumption by rats. Animal Learning \& Behavior, 1978, 6, 313-319.

Flaherty, C. F., Troncoso, B., \& Deschu, N. Open field behaviors correlated with reward availability and reward shift in three rat strains. American Journal of Psychology, 1979, 92, $385-400$.

Graham, J. M., \& Desjardins, C. Classical conditioning: Induction of luteinizing hormone and testosterone secretions in anticipation of sexual activity. Science, 1980, 210, 1039-1041.

Gutrman, N. Operant conditioning, extinction, and periodic reinforcement in relation to concentration of sucrose used as a reinforcing agent. Journal of Experimental Psychology, 1953, 46, 213-224.

Hulse, S. H. The case of the missing rule: Memory for reward vs. formal structure in serial-pattern learning by rats. Animal Learning \& Behavior, 1980, 8, 689-690.

Hulse, S. H., \& Dorsky, N. P. Serial pattern learning by rats: Transfer of a formally defined stimulus relationship and the significance of nonreinforcement. Animal Learning \& Behavior, $1979,7,211-220$.

Logan, F. A. Incentive. New York: Yale University Press, 1960.

Mackintosh, N. J., \& LoRD, J. Simultaneous and successive contrast with delay of reward. Animal Learning \& Behavior, 1973, 1, 283-286.

Pfaffman, C. The pleasures of sensation. Psychological Review, 1960, 67, 253-268.

SPEAR, N. E. The processing of memories: Forgetting and retention. Hillsdale, N.J: Erlbaum, 1978.

Vogel, J. R., Mikulka, P. J., \& Spear, N. E. Effects of shifts in sucrose and saccharin concentrations on licking behavior in the rat. Journal of Comparative and Physiological Psychology, $1968,66,661-666$.

Williams, B. A. Contrast, component duration, and the following schedule of reinforcement. Journal of Experimental Psychology: Animal Behavior Processes, 1979, 5, 379-396.

Williams, B. A. The following schedule of reinforcement as a fundamental determinant of steady state contrast in multiple schedules. Journal of the Experimental Analysis of Behavior, 1981, 35, 293-310.

Young, P. T., \& Madsen, C. H., JR. Individual isohedons in sucrose-sodium chloride and sucrose-saccharin gustatory areas. Journal of Comparative and Physiological Psychology, 1963, 56, 903-909.

(Manuscript received June 16, 1981; revision accepted for publication November $28,1981$. 